\title{
Ultramicroscopic Structures of the Leptomeninx of Mice with Communicating Hydrocephalus Induced by Human Recombinant Transforming Growth Factor- $\beta 1$
}

\author{
Junpei NITTA and Tsuyoshi TADA \\ Department of Neurosurgery, Shinshu University School of Medicine, Matsumoto, Nagano
}

\begin{abstract}
An experimental model of communicating hydrocephalus was developed based on intrathecal injection of human recombinant transforming growth factor- $\beta 1$ (hrTGF- $\beta 1$ ) in the mouse. To clarify the mechanism of this hydrocephalus model, the ultrastructure of the leptomeninx in the process of ventricular dilation was examined in C57/BL6 mice injected intrathecally with $60 \mathrm{ng}$ of hrTGF- $\beta 1$. The leptomeninx was examined at various periods after injection by light and electron microscopy. Immunostaining for fibroblasts and macrophages was also performed. Leptomeninx within a week after injection showed that the thin cytoplasmic processes of leptomeningeal cells formed a laminated structure with a meshwork, which was almost the same as the controls. In the second week, many cells with a round nucleus appeared in the leptomeninx. Immunohistochemically, these cells were positive for anti-fibroblast antibody and negative for anti-Mac-1 and anti-macrophage BM-8 antibodies. Three weeks later, the laminated structure was disrupted and abundant deposition of collagen fibers was found in the intercellular space of the leptomeninx. Such inter-meningeal fibrosis would disturb cerebrospinal fluid flow in the mouse leptomeninx and cause slowly progressive ventricular dilation.
\end{abstract}

Key words: transforming growth factor- $\beta 1$, communicating hydrocephalus, leptomeninx, mouse, fibroblast

\section{Introduction}

Patients with subarachnoid hemorrhage (SAH) sometimes develop communicating hydrocephalus in the chronic stage. The cerebrospinal fluid (CSF) pressure of such patients is usually "normal," a condition is well known as normal pressure hydrocephalus (NPH). Clinically, NPH is quickly improved by ventriculoperitoneal shunt surgery, but the pathogenesis has not been clarified.

We previously found significantly increased transforming growth factor- $\beta 1$ (TGF- $\beta 1$ ) level in the CSF immediately after $\mathrm{SAH}$, which remained higher in patients who subsequently developed hydrocephalus than in patients without hydrocephalus. ${ }^{5)}$ To study the role of TGF- $\beta 1$ in CSF after SAH, we experimentally injected human recombinant TGF- $\beta 1$ (hrTGF- $\beta 1$ ) into mice, and found that hrTGF- $\beta 1$ in- duced communicating hydrocephalus. ${ }^{\text {g) }}$

Experimental hydrocephalus can be induced in various animals by placing a small object at the outlets of ventricles or injecting foreign materials in the cistern. The inflammatory response of the leptomeninx obliterates the outlets of the fourth ventricle. Thus, almost all animal models of hydrocephalus are obstructive, and animal models of communicating hydrocephalus are very rare. ${ }^{1]}$

We have been developing this communicating hydrocephalus model in the mouse to study the pathogenesis of NPH after SAH. Here, we report the changes in light and electron microscopy features in the leptomeninx during the process of ventricular dilation. 


\section{Materials and Methods}

\section{Mice and reagents}

Pregnant C57/BL6 mice were purchased from SLC (Shizuoka), and 10-day-old offspring were used for this experiment. Mice delivered by the same mother were used as one group. Groups consisted of four to eight mice. hrTGF- $\beta 1$ was purchased from Austral Biologicals (San Ramon, Calif., U.S.A.).

\section{Intrathecal injection of TGF- $\beta 1$}

Mice were anesthetized with diethylether on the 10th day after birth. Thirty ng of hrTGF- $\beta 1$ in $30 \mu \mathrm{l}$ of phosphate-buffered saline (PBS), containing $0.1 \%$ bovine serum albumin (BSA) as a carrier protein, was injected into the right parietal head through a microsyringe so that the material leaked back and was distributed into the inter-meningeal CSF space. On the 11th day, the same material was injected into the left parietal head in the same way (Fig. 1). Tenday-old mouse skull is soft enough to be penetrated with a microsyringe. Non-treated age-matched mice, and mice injected with $30 \mu \mathrm{l}$ of PBS with BSA were used as controls.

\section{Preparation of specimens}

Mice were sacrificed at $3,5,7$, and 10 days, and 2, 3,6 , and 12 weeks after injection. PBS-injected mice and non-treated mice were also sacrificed on the same day. The head was sectioned coronally just caudal to the coronal suture. The whole brain was fixed with $10 \%$ neutral buffered formalin solution and embedded in paraffin.

\section{Microscopy studies}

Paraffin-embedded sections were observed under the light microscope with HE staining.

Specimens for electron microscopy were obtained from the frontal convexity region. The specimens were fixed in $2.5 \%$ glutaraldehyde and $1 \% \mathrm{OsO}_{4}$, and

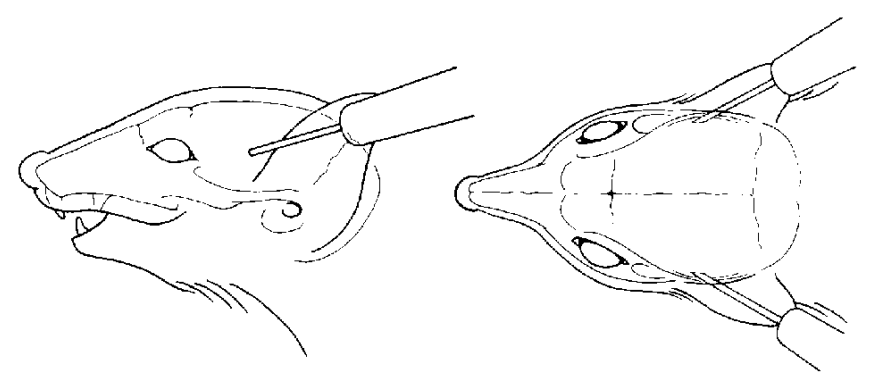

Fig. 1 Line drawing showing the sites of injection in the sagittal (left) and horizontal planes (right). embedded in Epon. Ultrathin sections were stained with uranyl acetate and lead citrate, and observed with a JEM-1200EX electron microscope $(80 \mathrm{kV})$ (Nippon Electric Co., Tokyo). The width of the leptomeninx was measured at the center of each electron micrograph.

\section{v. Immunohistochemical staining}

Deparaffinized slices obtained 1, 3, and 6 weeks after injection were incubated for 30 minutes with $1 \%$ normal rabbit serum, then incubated with rat antimouse fibroblast monoclonal antibody (MAb) or rat anti-Mac-1 MAb (Cedarlane Laboratories, Ontario, Canada) or rat anti-macrophage BM-8 MAb (BMA Biomedicals, Augst, Switzerland) for 3 hours at room temperature. All antibodies were diluted 100 times. After washing, fluorescein isothiocyanateconjugated rabbit anti-rat $\mathrm{G} \mathrm{Fab}$ fragment (Serotec, Oxford, England) was applied for 1 hour at room temperature. The penetrated region of the brain 1 week after injection and subcutaneous tissue were used as positive controls, and specimens not incubated with the first antibody was used as the negative control.

\section{Results}

\section{Light microscopic features of leptomeninx of TGF- $\beta 1$-injected mouse}

Figure 2 shows the light micrographs of the leptomeninx of mice at $1,3,6$, and 12 weeks after injection of hrTGF- $\beta 1$. The leptomeninx within a week after injection consisted of several layers of leptomeningeal cells, which was essentially the same as that of the PBS-injected and non-treated mice at all periods. The leptomeninx from 2 to 3 weeks after injection showed accumulation of many cells with a round nucleus and thickening. Similar findings were observed in the leptomeninx at 6 and 12 weeks.

\section{Ultramicroscopic structure of normal mouse leptomeninx}

Normal mouse leptomeninx consisted of several tiers of some flattened leptomeningeal cells. The width of the leptomeninx near the middle cerebral artery was $6.1 \pm 1.0 \mu \mathrm{m}(\mathrm{n}=6)$ as measured in the electron micrographs. The leptomeningeal cells had very thin and loosely arranged cytoplasm containing abundant mitochondrias. The processes of the cytoplasm were connected by tight junctions, but the intercellular spaces were sufficiently wide. Collagen fibers were sparse in the intercellular space. A basement membrane under the single leptomeningeal cell layer clearly separated the leptomeninx from the brain parenchyma (Fig. 3). 


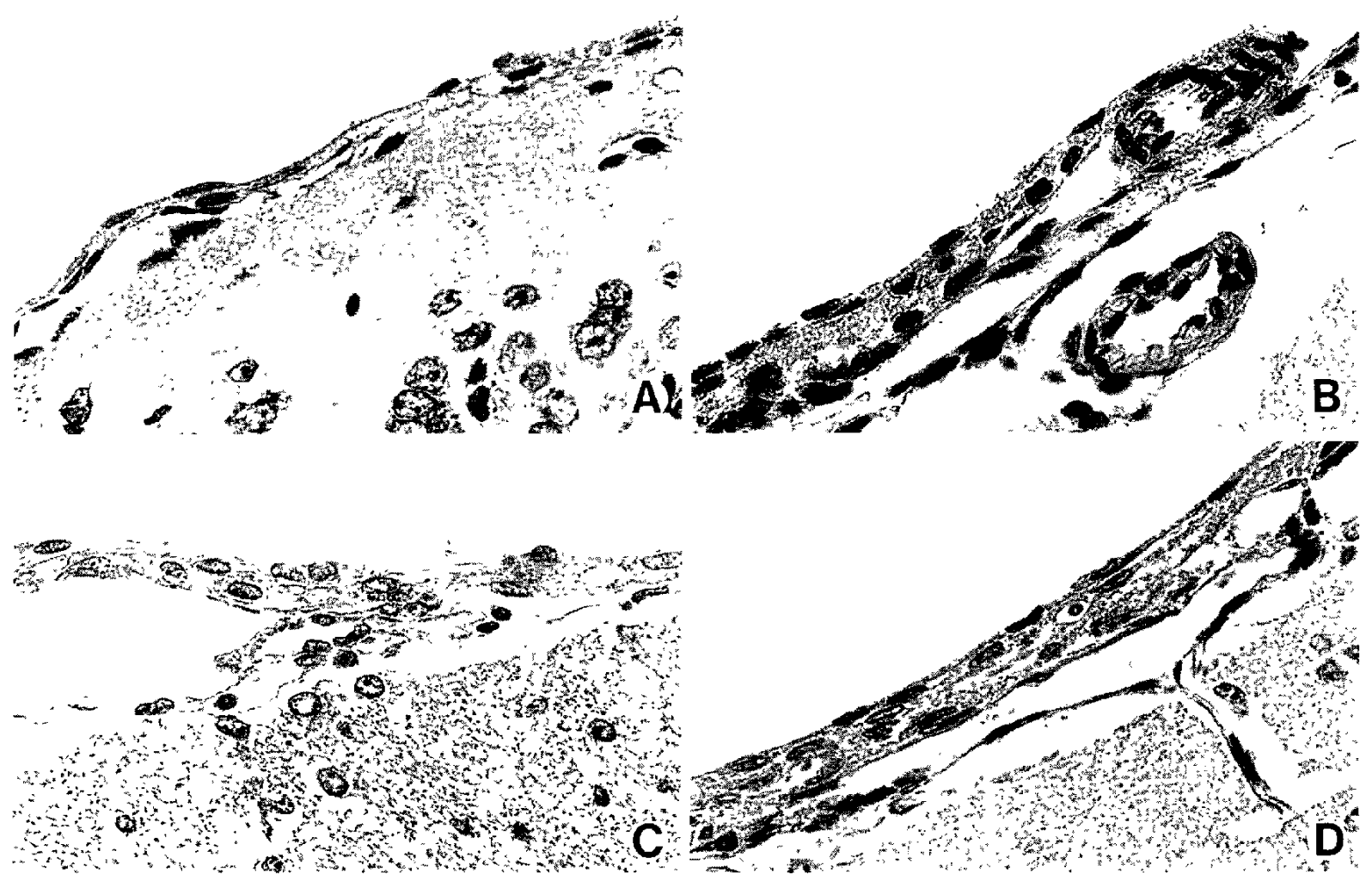

Fig. 2 Light micrographs showing the leptomeninx of the mouse at various periods after transforming growth factor- $\beta 1$ injection. At 1 week (A), the leptomeninx consists of several layers of leptomeningeal cells. At 3 (B), 6 (C), and 12 weeks (D), cells with a round nucleus have accumulated in the leptomeninx, and the leptomeninx has thickened. HE stain, $\times 200$.

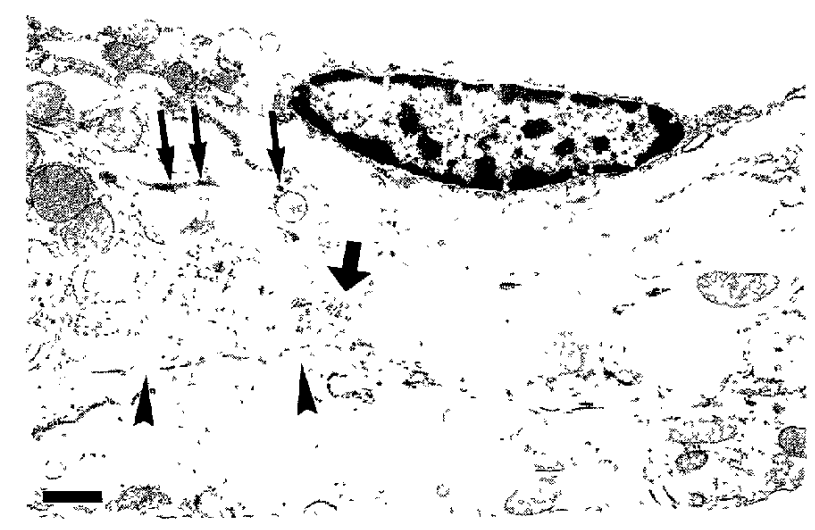

Fig. 3 Electron micrograph of normal mouse leptomeninx. The leptomeningeal cells have very thin cytoplasm, and the processes of the cytoplasm compose a loose meshwork. Tight junctions between the processes (arrows), basement membrane (arrowheads), and collagen fiber (thick arrow) are seen. $\operatorname{Bar}=1 \mu \mathrm{m}$.
III. Ultramicroscopic changes of leptomeninx after TGF- $\beta 1$ injection

The leptomeningeal structure at $3(\mathrm{n}=7), 5(\mathrm{n}=$ $6)$, and $7(n=5)$ days after TGF- $\beta 1$ injection was almost the same as that of controls, showing that the laminated structure of the leptomeninx was well preserved (Fig. 4).

The leptomeninx at 10 days after injection of TGF$\beta 1(n=4)$ showed cells with a round nucleus and various sizes of electron dense vesicles in the cytoplasm between the leptomeningeal layers. Fourteen days after injection $(\mathbf{n}=5)$, the cells increased in number, and the leptomeninx was thickened by the cell accumulation to $8.7 \pm 1.5 \mu \mathrm{m}(\mathrm{n}=5)$. The laminated structure of leptomeninx was still observed. A few collagen fibers were found between the leptomeningeal cells (Fig. 5). The leptomeninx of mice 10 days after PBS injection showed a small number of cells with electron dense vesicles in the cytoplasm, but they decreased in number until 14 days after injection, and the leptomeningeal laminated structure was well preserved. Such cells were not found in non-treated mice. 


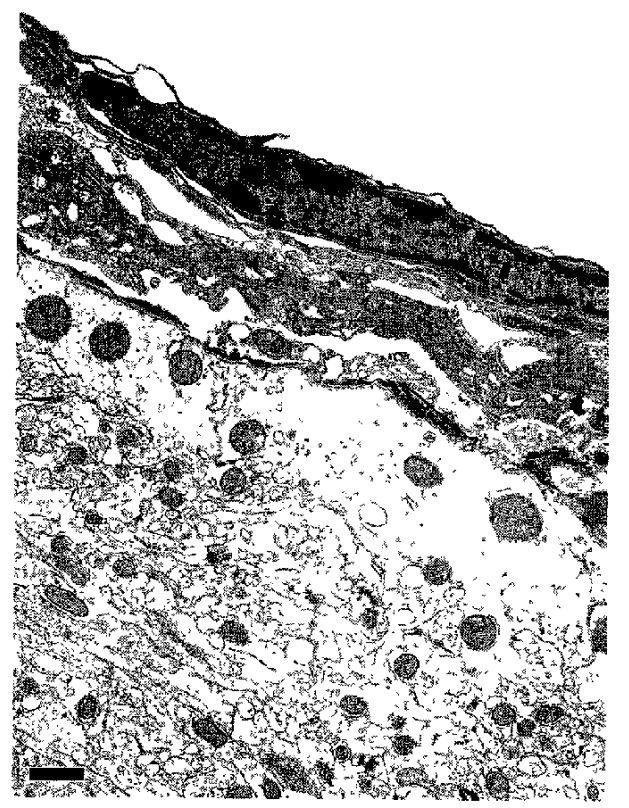

Fig. 4 Electron micrograph of leptomeninx 5 days after transforming growth factor- $\beta 1$ injection. The laminated structure is preserved. Bar $=1 \mu \mathrm{m}$.

Three weeks after TGF- $\beta 1$ injection $(n=4)$, the thickness of the leptomeninx had become $10.8 \pm$ $1.8 \mu \mathrm{m}(\mathrm{n}=4)$ with increased cellularity. The laminated structure was obscured, and the number of mitochondrias in the cytoplasm had decreased. Col- lagen fibers were abundant, and the intercellular space of the leptomeninx was obscured. The accumulating cells became compact in shape, and the

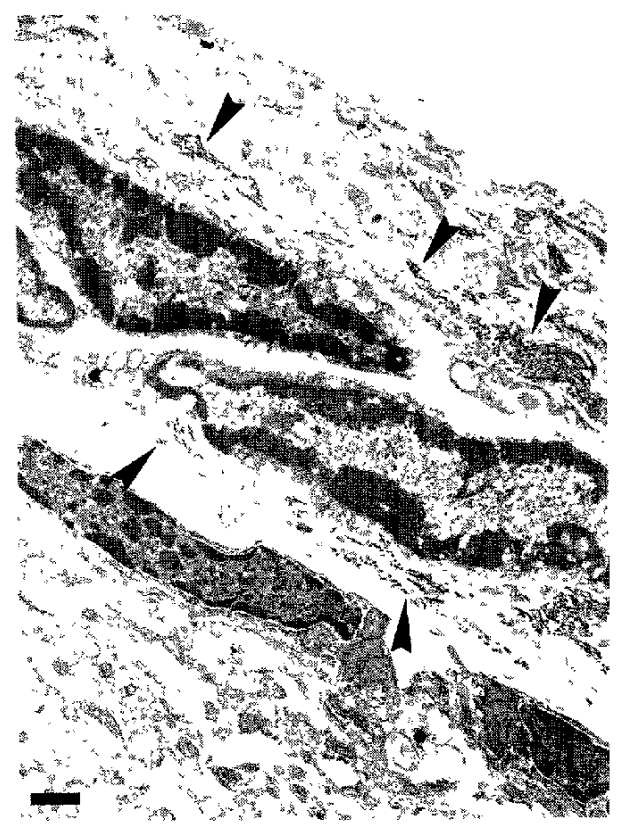

Fig. 6 Electron micrograph of leptomeninx at 3 weeks after transforming growth factor- $\beta 1$ injection. The laminated structure is disrupted. The intercellular space of the leptomeninx is tight and abundant collagen fibers (arrowheads) are observed. Bar $=1$ $\mu \mathrm{m}$.

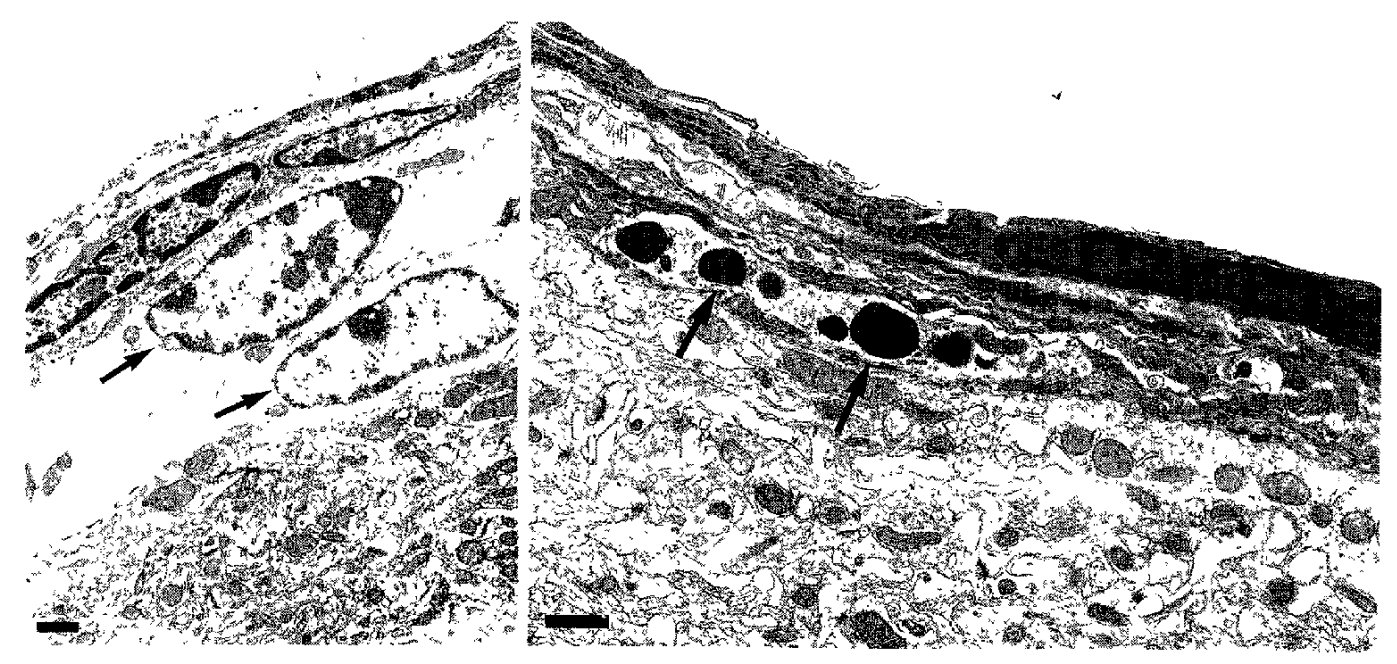

Fig. 5 Electron micrographs of leptomeninx at 2 weeks after transforming growth factor- $\beta 1$ injection. left: The cells with a round nucleus are present in the leptomeninx (arrows), and are different from leptomeningeal cells with a flattened nucleus. right: Various sizes of electron dense vesicles (arrows) are seen in the cytoplasm of the accumulating cells. Bar $=1 \mu \mathrm{m}$. 

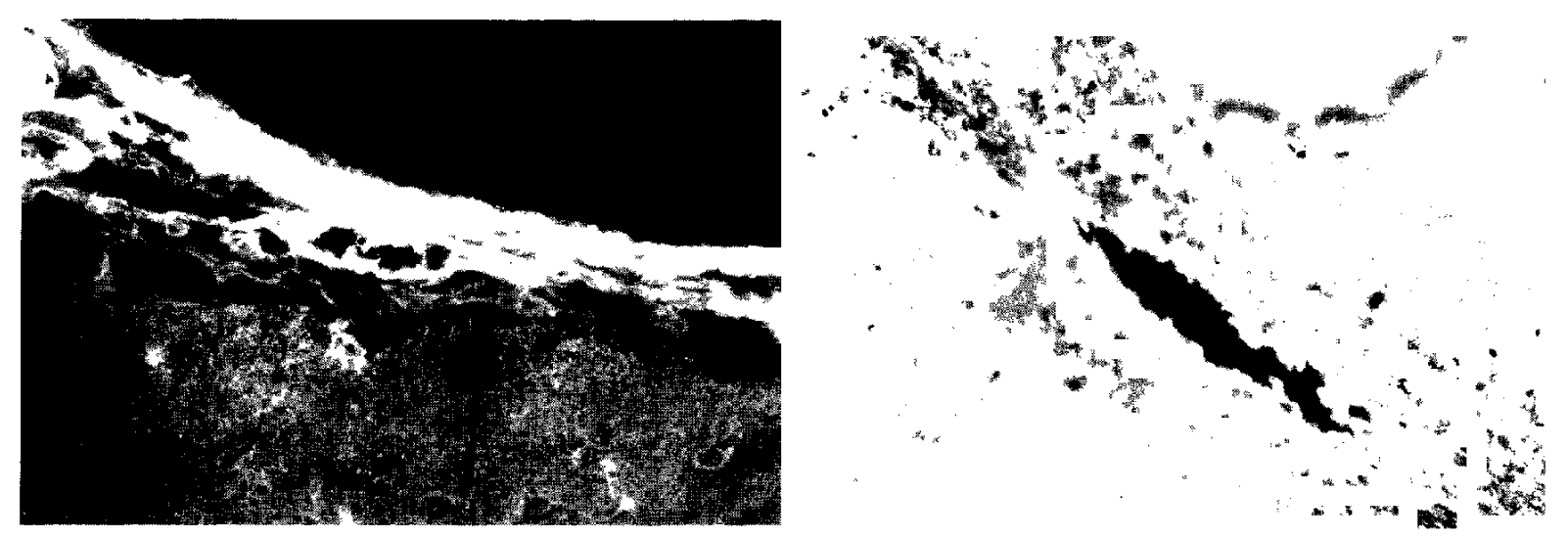

Fig. 7 Light micrographs of immunohistochemical staining for anti-fibroblast monoclonal antibody. left: Cells in the leptomeninx at 3 weeks after transforming growth factor- $\beta 1$ injection are positive for anti-fibroblast antibody, right: Mouse brain of the penetrated portion at 1 week after injection contains many anti-fibroblast antibody-positive cells. $\times 200$.

number of electron dense vesicles in the cytoplasm decreased (Fig. 6). In contrast, the laminated structure of the leptomeningeal cells and intercellular space was preserved in non-treated and PBS-injected mice.

\section{Immunohistochemical findings}

The accumulated cells in the leptomeninx 1,3 , and 6 weeks after injection of TGF- $\beta 1$ were negative for BM-8 nor Mac-1 MAb, and were positive for antifibroblast MAb (Fig. 7 left). No anti-fibroblast antibody-positive cells were found in the leptomeninx of non-treated mice. Accumulated cells at the penetrated region of the brain 1 week after injection of TGF- $\beta 1$ were also positive for anti-fibroblast $\mathrm{MAb}$ (Fig. 7 right).

\section{Discussion}

The mouse leptomeninx consists of loosely arranged leptomeningeal cells which contact each other with extremely elongated cytoplasmic processes, so forming a loose meshwork. CSF circulates through the intercellular space of the leptomeningeal cells. ${ }^{6}$ CSF mainly drains into the nasal lymphatic pathway in the mouse instead of vessels through the arachnoid villi as in humans. ${ }^{4}$

The TGF- $\beta 1$-induced hydrocephalus in the present mouse model is communicating; we previously showed the absence of obstruction at the outlets of ventricles and ventricular reflux of ink injected into the parietal inter-meningeal CSF space of the hydrocephalic mouse ${ }^{3,91}$ In contrast to the kaolin-injected models of hydrocephalus, there are no inflammatory cells which react to the foreign material around the outlet of ventricles in the hydrocephalic mouse. ${ }^{10)}$

This mouse model of communicating hydrocephalus is slowly progressive. The increase in body weight of the TGF- $\beta 1$-injected mouse starts to reverse 3 weeks later, and ventricular dilation becomes obvious at 6 weeks after injection, but is not accompanied by cranial enlargement. Therefore, the intracranial pressure of the mouse is not so elevated and ventricular dilation requires a long time to develop. ${ }^{9]}$

In this study, light microscopy revealed increased cellularity and thickened leptomeninx over 3 weeks after TGF- $\beta 1$ injection. The morphological changes of the leptomeninx in the process of ventricular dilation were investigated within 3 weeks after injection. The ultrastructural changes of leptomeninx in the process of ventricular dilation could be divided into three periods. Within a week, there were no histological changes in the leptomeninx. In the second week, many cells with a round nucleus and various sizes of dense vesicles in the cytoplasm accumulated in the leptomeninx. Leptomeningeal macrophages and fibroblasts in the mouse have very similar ultrastructural characteristics to the cells containing various size of electron dense vesicles. ${ }^{81}$ Immunohistochemical examination of the cells showed that they were fibroblasts. Three weeks after injection, abundant collagen fibers were secreted in the intercellular space of the leptomeninx.

TGF- $\beta 1$ stimulates the proliferation of fibroblasts ${ }^{71}$ and also stimulates fibroblasts to secrete several types of collagen. ${ }^{21}$ Consequently, the intrathecal injection of hrTGF- $\beta 1$ is considered to facilitate the accumulation of fibroblasts and the fibroblasts secrete 
the collagen in the leptomeninx. Such deposition of collagen would disturb the CSF flow in the intermeningeal space, this resulting in slowly progressive ventricular dilation.

We suggest that further study of the exogenous TGF- $\beta 1$ injected in the inter-meningeal CSF space of mouse will help to clarify the pathogenesis of communicating hydrocephalus after SAH.

\section{Acknowledgment}

This study was supported by grant 20 (1996) from the Japan Spina Bifida \& Hydrocephalus Research Foundation.

\section{References}

1) Hochwald GM: Minireview. Animal models of hydrocephalus: Recent development. Proc Soc Exp Biol Med 178: 1-11, 1985

2) Ignotz RA, Massague J: Transforming growth factorbeta stimulates the expression of fibronectin and collagen and their incorporation into the extracellular matrix. J Biol Chem 261: 4337-4345, 1986

3) Kanaji M, Tada T, Kobayashi S: A murine model of communicating hydrocephalus: role of TGF- $\beta 1$. J Clin Neurosci 4: 51-56, 1997

4) Kida S, Pantazis A, Weller RO: CSF drains directly from the subarachnoid space into nasal lymphatics in the rat. Anatomy, histology and immunological significance. Neuropathol Appl Neurobiol 19: 480-488, 1993

5) Kitazawa K, Tada T: Elevation of transforming growth factor- $\beta 1$ level in cerebrospinal fluid of patient suffering hydrocephalus following subarachnoid hemorrhage. Stroke 25: 1400-1404, 1994

6) Oda Y, Nakanishi I: Ultrastructure of mouse leptomeninx. J Comp Neurol 225: 448-457, 1984

7) Postlethwaite AE, Keski-Oja J, Moses HL, Kang AH: Stimulation of the chemotactic migration of human fibroblasts by transforming growth factor $\beta$. J Exp Med 165: 251-256, 1987

8) Sturrock RR: An ultrastructural study of the development of leptomeningeal macrophages in the mouse and rabbit. J Anat 156: 207-215, 1988

9) Tada T, Kanaji M, Kobayashi S: Induction of communicating hydrocephalus in mice by intrathecal injection of human recombinant transforming growth factor- $\beta 1$. J Neuroimmunol 50; 153-158, 1994

10) Yamada H, Yokota A, Haratake J, Horie A: Morphological study of experimental syringomyelia with kaolin-induced hydrocephalus in a canine model. J Neurosurg 84: 999-1005, 1996

Address reprint requests to: J. Nitta, M.D., Seguchi Neu- rosurgical Hospital, 218-2 Kuroda, Kamisato, Iida, Nagano 395-0004, Japan.

\section{Commentary}

Using an experimental murine model of communicating hydrocephalus produced by intrathecal injection of hrTGF- $\beta 1$, the authors studied the pathogenesis of normal pressure hydrocephalus (NPH) after subarachnoid hemorrhage (SAH). They examined changes in the features of the leptomeninx during the process of ventricular dilatation using both light and electron microscopy. On the basis of their histological observations, they considered that disturbance of the CSF flow in the intermeningeal space caused by deposition of collagen results in slowly progressive hydrocephalus. Assuming that the results obtained in mice can be extrapolated to humans, this article contains important findings that shed light on the pathogenesis of communicating hydrocephalus after $S A H$, and may help to establish a new therapy for NPH. However, further study will be necessary using higher mammals with a CSF absorption pathway similar to that in humans. I am not sure whether the expression "round nucleus" is suitable for describing the leptomeningeal cells after hrTGF- $\beta 1$ injection. Their shape seems to be rather ovoid with an elongated longitudinal axis, rather than round.

Kouzo MortTake, M.D. Department of Neurosurgery Shimane Medical University Shimane, Japan

The authors have performed an interesting study in which communicating hydrocephalus has been created in mice following the intracerebral injection of TGF- $\beta 1$. The impetus to develop this model stemmed from their prior work in which it was observed that patients with subarachnoid hemorrhage who went on to develop hydrocephalus frequently had higher CSF levels of TGF- $\beta$ than did controls.

In the present study, light and electron microscopic evidence has shown that TGF- $\beta 1$ injections lead to a thickening in the leptomeninx by 3 years post injection. This thickening was the result of the deposition of abundant amounts of collagen fibers in the leptomeninx. Ultimately, the thickened leptomeninx leads to poor CSF diffusion and progressive hydrocephalus.

There is great interest currently in the pleotrophic effects of TGF- $\beta$ in human disease. TGF- $\beta$ is known to be an immunosuppressant in the central nervous system, a regulator of cell cycle control, and a collaborator in several developmental signaling pathways. I congratulate the authors in their development of this 
model of hydrocephalus. In future studies, I hope they will be able to determine the mechanism by which collagen deposition is stimulated, and what type of collagen is laid down.

James T. RUTKA, M.D., F.R.C.S.C. Division of Neurosurgery The Hospital for Sick Children Brain Tumor Research Laboratory University of Toronto Ontario, Canada

This article described immunohistochemical as well as ultramicroscopic findings of an experimental model of communicating hydrocephalus which was developed by the authors. This study mainly considered the pathological changes in the meningeal membrane resulting from the intrathecal injection of hrTGF- $\beta 1$. Rich collagen fibers were demonstrated in the leptomeninx which were supposed to be formed by fibroblast proliferation stimulated by hrTGF- $\beta 1$.

The unique point of this experimental model is thought to be the slowly progressive manner of the ventricular dilatation. In contrast to the kaolin-injec- tion model with acute hydrocephalus, this nature of progress may mimic the clinical situation of postsubarachnoid hemorrhage or post-inflammatory hydrocephalus which is often encountered by neurosurgeons. The transgenic mouse congenital hydrocephalus model cannot mimic this point.

From the therapeutic view point, further study regarding the reversibility of hydrocephalus in this model, in both ventricular size and pathohistological change is required. It should be noticed that the meningeal membrane secretes a lot of unknown proteins into the cerebrospinal fluid. Increased numbers of fibroblasts may induce mechanical disturbance of the cerebrospinal fluid flow but may also potentially change the physiological status in cerebrospinal fluid absorption, which is known to be an additional factor in the development of hydrocephalus.

Norihiko TAMAKI, M.D. and Takeshi KONDOH, M.D. Department of Neurosurgery Kobe University School of Medicine Kobe, Japan 